\title{
High Pulse Energy, Rep. Rated Nd:glass Laser with Wavefront Correction
}

\author{
Ryo YASUHARA, ${ }^{1}$ Takashi SEKINE, ${ }^{1}$ Takashi KURITA, ${ }^{1}$ Tadashi IKEGAWA, ${ }^{1}$ Osamu MATSUMOTO, ${ }^{1}$ \\ Toshiyuki KAWASHIMA, ${ }^{1}$ Masahiro MIYAMOTO, ${ }^{1}$ Hirofumi KAN, ${ }^{1}$ Hidetsugu YOSHIDA, ${ }^{2}$ Junji \\ KAWANAKA, ${ }^{2}$ Masahiro NAKATSUKA, ${ }^{2}$ Yasukazu IZAWA, ${ }^{2}$ and Tadashi KANABE ${ }^{3}$ \\ ${ }^{1}$ Hamamatsu Photonics K. K., 5000, Hirakuchi, Hamakita-ku, Hamamatsu, Shizuoka 434-8601 \\ ${ }^{2}$ Institute of Laser Engineering, Osaka University, 2-6, Yamada-oka, Suita, Osaka 565-0871 \\ ${ }^{3}$ Graduate School of Engineering, University of Fukui, 3-9-1, Bunkyou, Fukui 910-8507
}

(Received February 18, 2008)

\begin{abstract}
We report a high average power and high pulse energy diode-pumped Nd:glass master oscillator power amplifier system that is consisted of a thermally-edge-controlled zigzag slab amplifier and stimulated Brillouin scattering (SBS) mirror for use of pumping source of ultra intense laser system. This phase conjugated system produces an average power of $213 \mathrm{~W}$ at $10 \mathrm{~Hz}$ in 8.9-ns pulse (2.4 GW peak power) with an optical-to-optical conversion efficiency of $11.7 \%$ and a near diffraction limited beam quality. To the best of our knowledge these results represent the highest average power achieved from a Nd:glass based laser amplifier system.
\end{abstract}

Key Words: Diode-pumped Lasers, Phase conjugate, High average power laser.

\section{Introduction}

Recently, pulse lasers with both of high pulse energy and high average power, are strongly desired for various industrial and scientific applications such as laser peening, ${ }^{1)}$ laser processing, ${ }^{2)}$ high-energy particle generation, ${ }^{3)}$ hard $\mathrm{x}$-ray generation, ${ }^{4)}$ inertial fusion energy (IFE) ${ }^{5,6)}$ and so on. For such applications, it is desirable for driver laser to extract a large stored energy with good beam quality and high overall efficiency from a single amplifier aperture. A diode-pumped solid-state laser (DPSSL) based on Nd:glass gain medium has possibility for these requirements. A diode-pumping complements solid-state lasers in overall efficiency, long life time of pumping source, performance stability and system reliability. And the Nd:glass gain medium have a availability in large size and a moderate emission cross section (3.5 $\mathrm{x}$ $10-20 \mathrm{~cm}^{2}$ ) suited to the large stored energy for high energy per pulse operation, however it exhibits a lower thermal characteristics for high average power operation. In particular, thermal wavefront distortion that is critical issue in high average power operation is strongly appeared in Nd;glass gain medium.

One of a solution to compensate thermal wavefront aberration is thermally-edge-controlled zig-zag slab (TECS) laser amplifier with SBS mirror. Thermal wavefront distortion parallel to zig-zag direction automatically compensated by zig-zag optical pass. Although many papers are reported the potential of zig-zag slab laser configuration and demonstrated the several kilowatt average power by this configuration, high energy pulse operation with high over roll efficiency has not demonstrated. TECS techniques that is consisted by actively heated edge claddings on the top and bottom of the laser slab to flatten the temperature gradient suppress a thermal effects perpendicular to the zig-zag averaging propagation. It was reported in our previous work. ${ }^{7)}$ A SBS mirror compensates the high order aberration in the laser slabs. A notable examples are reports of high beam quality of $100 \mathrm{~W}$ laser system using a Nd:YAG DPSSL system and a $150 \mathrm{~W}$ flash lamp pumped Nd:glass system. ${ }^{8,9)}$

In this report, we have demonstrated $21.3 \mathrm{~J}$ laser pulse in 8.9 ns pulse duration at $10 \mathrm{~Hz}$ repetition rate with $11.7 \%$ optical to optical (o-o) efficiency. To our knowledge, this laser obtained the highest average power from single aperture of a laser amplifier based glass gain medium with good beam quality and high conversion efficiency.

\section{Laser system architecture}

The concept of our Laser system is based on HALNA (High Average-power Laser for Nuclear Fusion Application) project at ILE, Osaka University. ${ }^{5)}$ This laser system consist of

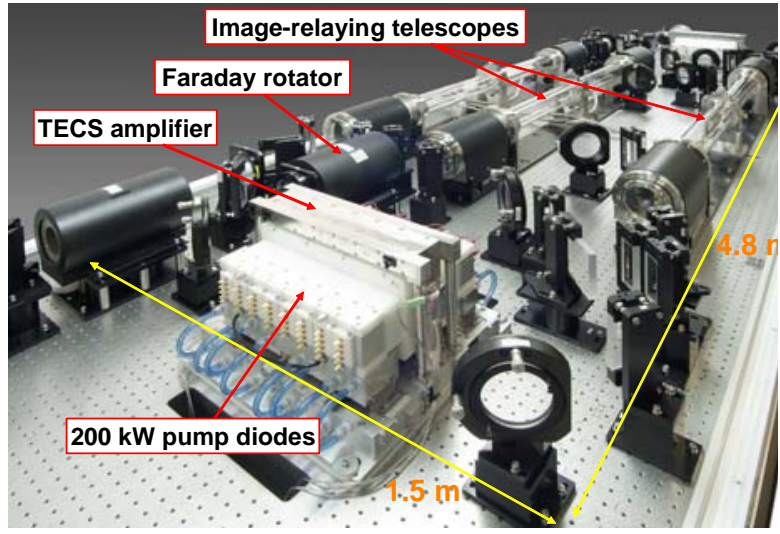

Fig. 1 A photograph of diode-pumped Nd:glass zig-zag slab laser sytem with TECS technology and SBS mirror. 
$\mathrm{Nd}$ :glass gain medium, diode pumping, water cooling, zig-zag optical path, a master oscillator - power amplifier (MOPA) system and multi-pass amplifier architecture, dual TECS amplifier with zig-zag multi optical pass and SBS mirror.

Figure 1 shows a photograph of diode-pumped Nd:glass zig-zag slab laser system with TECS technology and SBS mirror. Front-end of the system is a combination of a $1053 \mathrm{~nm}$, Yb-doped, distributed-feedback fiber pulsed oscillator and a diode-pumped Nd:YLF regenerative ring amplifier. The laser pulse amplified by front-end is optically expand to the full aperture of a slab amplifier by the anamorphic telescope, and it is cut out by the rectangular serrated aperture of $0.8 \mathrm{~cm}$ wide $\mathrm{x}$ $4.8 \mathrm{~cm}$ high. The beam image at this point is relayed the slab amplifiers by use of the vacuum telescopes with a spatial filter, and subsequently it passes the slabs four times each in two symmetrical paths. Finally the output pulse is ejected by a thin-film polarizer. A double pass of the $45^{\circ}$ Faraday rotator provides a passive $90^{\circ}$ polarization rotation, and its cross-polarizing scheme compensates for thermally induced birefringence in the slabs. An adaptive wave-front correction by using SBS has successfully installed into the system.

\section{Result and discussion}

Figure 2 shows the extracted laser output energy from the laser system at $10 \mathrm{~Hz}$ repetition rate operation. The maximum output energy of $21.3 \mathrm{~J}$ was obtained with o-o efficiency of $11.7 \%$, when the input pulse energy was $125 \mathrm{~mJ}$. The extraction efficiency was $33.2 \%$ under the diode pump energy of $182 \mathrm{~J}$ (peak intensity of $800 \mathrm{~kW}$, pump wavelength of 803 $\mathrm{nm}$, pump duration of $200 \mu \mathrm{s}$ ). Waveform of laser output at $21.3 \mathrm{~J}$ is shown in Fig. 3. 8.9 ns (FWHM) pulse duration is observed and then peak power of laser output was $2.4 \mathrm{GW}$. The far field pattern (FFP) of output beam at $21.3 \mathrm{~J}$ is shown in the Fig. 4 (a). The energy occupation was calculated as the total intensity in the encircled pixels to that of the entire pixels in the far-field image. Over $60 \%$ of the entire energy was focused in 2 times of the diffraction limit (TDL) spot. Figure 4



Fig. 2. Laser output performance of output energy and extraction efficiency.

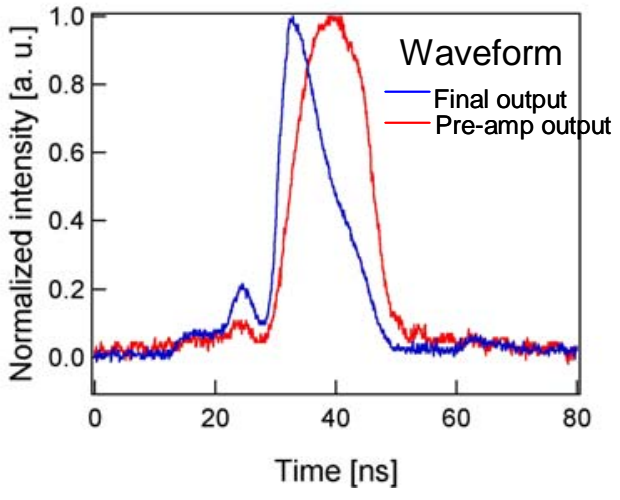

Fig. 3. Waveform of laser output and from pre- amplifier at $21.3 \mathrm{~J}$ x $10 \mathrm{~Hz}$.

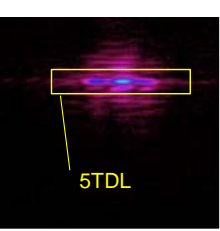

(a)

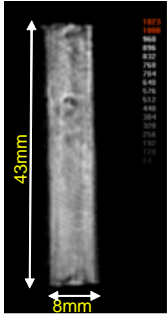

(b)
Fig. 4. (a) The far field pattern (FFP) of output beam at $21.3 \mathrm{~J}$. (b) The near field beam image at $21.3 \mathrm{~J}$ laser output.

(b) shows the near field beam image at $21.3 \mathrm{~J}$ laser output. The spatial profile of intensity in NFP is as uniform as $55 \%$ of filling factor, which was defined as the ratio of the average intensity in the encircled area to the peak intensity of the beam profile. In addition, average fluence of laser output was 6.2 $\mathrm{J} / \mathrm{cm} 2$.

We have demonstrated the $21.3 \mathrm{~J}$ in $8.9 \mathrm{~ns}$ at $10 \mathrm{~Hz}$ with $11.7 \%$ o-o efficiency. To our knowledge, this laser obtained the highest average power from single aperture of the glass gain medium based laser amplifier.

\section{Conclusion}

In conclusion, we have activated diode-pumped Nd:glass zig-zag slab laser. Average power of $213 \mathrm{~W}(21.3 \mathrm{~J}$ at $10 \mathrm{~Hz})$ was successfully achieved. The diffraction limited focusability was achieved by use of SBS phase conjugator. These results have indicated the potential of diode-pumped $\mathrm{Nd}$ :glass laser system with SBS mirror for high pulse energy and high average power laser driven industrial and scientific applications.

\section{Acknowledgements}

Authors thank Dr. Y. Tsuchiya at Hamamatsu Photonics K. $\mathrm{K}$. for his great contribution on this project, who is now deceased.

This work is partly supported by NEDO (New Energy and Industrial Technology Development Organization) under the Ministry of Economy, Trade and Industry in Japan. 


\section{References}

1) M. Korsunsky, J. Liu, D. Laundy, M. Golshan, and K. Kim: J. Strain Analysis 41 (2006) 113.

2) D. Ashkenasi, A. Rosenfeld, H. Varel, M. Wähmer and E. E. B. Campbell: Appl. Surf. Sci. 120 (1997) 65.

3) M. I. K. Santala, M. Zepf, F. N. Beg, E. L. Clark, A. E. Dangor, K. Krushelnick, M. Tatarakis, I. Watts, K. W. D. Ledingham, T. McCanny, et al: Appl. Phys. Lett. 78 (2001) 19.

4) J. D. Kmetec, C. L. Gordon, III, J. J. Macklin, B. E. Lemoff, G. S. Brown, and S. E. Harris: Phys. Rev. Lett. 68 (1992) 1527.
5) T. Kawashima, T. Kanabe, H. Matsui, E. Eguchi, M. Yamanaka, Y. Kato, M. Nakatsuka, Y. Izawa, S. Nakai, T.Kanzaki and H. Kan: Jpn. J. Appl. Phys. 40 (2001) 6415.

6) W.F. Krupke: Fusion Technol. 15 (1989) 377.

7) T. Kurita et al.: Conf. Laser and Electro Optics, Baltimore, USA, May 22-27, 2005 (Optical Society of America, Washington, D.C., 2005) CMJ5.

8) H. J. Eichler, A. Haase, and R. Menzel: IEEE J. Quant. Electron. 31 (1995) 1265.

9) C.B. Dain, L. E. Zapata, W. A. Neuman, M.A. Norton and L. A. Hackel: IEEE J. Quant. Electron. 31 (1995) 148. 